Published in final edited form as:

Amino Acids. 2020 September ; 52(9): 1319-1335. doi:10.1007/s00726-020-02894-5.

\title{
Intermittent leucine pulses during continuous feeding alters novel components involved in skeletal muscle growth of neonatal pigs
}

\author{
Rodrigo Manjarín ${ }^{1,2}$, Claire Boutry-Regard ${ }^{1}$, Agus Suryawan ${ }^{1}$, Angela Canovas ${ }^{3}$, Brian D. \\ Piccolo ${ }^{4,5}$, Magdalena Maj ${ }^{6}$, Mohammed Abo-Ismail' ${ }^{2}$, Hanh V. Nguyen ${ }^{1}$, Marta L. Fiorotto', \\ Teresa A. Davis ${ }^{1}$ \\ ${ }^{1}$ Children's Nutrition Research Center, Department of Pediatrics, Baylor College of Medicine, U.S. \\ Department of Agriculture/Agricultural Research Service, Houston, TX 77030, USA \\ ${ }^{2}$ Animal Science Department, California Polytechnic State University, 1 Grand Ave, San Luis \\ Obispo, CA 93407-0255, USA \\ ${ }^{3}$ Ontario Agricultural College, University of Guelph, Guelph, Canada \\ ${ }^{4}$ Arkansas Children's Nutrition Center, U.S. Department of Agriculture/Agricultural Research \\ Service, Little Rock, AR 72202, USA \\ ${ }^{5}$ Department of Pediatrics, University of Arkansas for Medical Sciences, Little Rock, AR 72202, \\ USA \\ ${ }^{6}$ Biological Sciences Department, California Polytechnic State University, San Luis Obispo, CA \\ 93407, USA
}

\section{Abstract}

When neonatal pigs continuously fed formula are supplemented with leucine pulses, muscle protein synthesis and body weight gain are enhanced. To identify the responsible mechanisms, we combined plasma metabolomic analysis with transcriptome expression of the transcriptome and protein catabolic pathways in skeletal muscle. Piglets $(n=23,7$-day-old $)$ were fed continuously a milk replacement formula via orogastric tube for 21 days with an additional parenteral infusion $\left(800 \mu \mathrm{mol} \mathrm{kg}^{-1} \mathrm{~h}^{-1}\right.$ ) of either leucine (LEU) or alanine (CON) for $1 \mathrm{~h}$ every $4 \mathrm{~h}$. Plasma metabolites were measured by liquid chromatography-mass spectrometry. Gene and protein expression analyses of longissimus dorsi muscle were performed by RNA-seq and Western blot,

\footnotetext{
${ }^{\circledR}$ Rodrigo Manjarín, rmanjari@ calpoly.edu.

Author contributions Conceptualization-CBR, MF and TD. Data curation-CBR, AS and HN. Formal analysis-RM, BP, AC, MM, and MI. Funding acquisition-MF and TD. Investigation-CBR, AS, HN, MF and TD. Methodology-CBR, AS and TD. Project administration-MF and TD. Resources-MF and TD. Supervision-MF and TD. Validation-MF and TD. VisualizationRM, BP, AC, MM, and MI. Writing—original draft—RM, BP, AC, MM, and MI. Writing—review and editing—all authors.

Data availability Raw data are provided as online resources.

Conflict of interest The authors declare that they have no conflict of interest.

Ethics approval Experiments were carried out in accordance with the Institutional Animal Care and Use Committee of Baylor College of Medicine and conducted in accordance with the National Research Council's Guide for the Care and Use of Laboratory Animals (2011).
}

Electronic supplementary material The online version of this article (https://doi.org/10.1007/s00726-020-02894-5) contains supplementary material, which is available to authorized users. 
respectively. Compared with CON, LEU pigs had increased plasma levels of leucine-derived metabolites, including 4-methyl-2-oxopentanoate, beta-hydroxyisovalerate, $\beta$ -

hydroxyisovalerylcarnitine, and 3-methylglutaconate $(P \leq 0.05)$. Leucine pulses downregulated transcripts enriched in the Kyoto Encyclopedia of Genes and Genomes terms "spliceosome," "GAP junction," "endocytosis," "ECM-receptor interaction," and "DNA replication". Significant correlations were identified between metabolites derived from leucine catabolism and muscle genes involved in protein degradation, transcription and translation, and muscle maintenance and development $(P \leq 0.05)$. Further, leucine pulses decreased protein expression of autophagic markers and serine/threonine kinase 4 , involved in muscle atrophy $(P \leq 0.01)$. In conclusion, results from our studies support the notion that leucine pulses during continuous enteral feeding enhance muscle mass gain in neonatal pigs by increasing protein synthetic activity and downregulating protein catabolic pathways through concerted responses in the transcriptome and metabolome.

\section{Keywords}

Leucine; Orogastric feeding; Protein synthesis; Transcriptomics; Metabolomics

\section{Introduction}

Pediatric enteral nutrition via oro- or nasogastric feeding is indicated when nutrient requirements cannot be achieved by normal food intake in patients with a functional gut, such as preterm infants with uncoordinated sucking and swallowing, and children with protein intolerance or Crohn's disease (Hill et al. 1995; Dziechciarz et al. 2007; Braegger et al. 2010). While objective criteria for pediatric enteral nutrition has been characterized, the best method of delivery (i.e., continuous vs. intermittent feeding) is still unclear (Braegger et al. 2010). Compared with continuous feeding, intermittent bolus feeding is thought to be more physiological because it promotes a cyclical pattern of release of gastrointestinal hormones as normally seen in healthy term infants, which may improve metabolic homeostasis, as well as gastrointestinal tract development and function (Aynsley-Green et al. 1982; Mashako et al. 1987; Shulman et al. 1994). In addition, we have shown that intermittent bolus feeding induces a pulsatile pattern of circulating amino acids (AA) and insulin, which activates AA and insulin signaling pathways in skeletal muscle to enhance protein synthetic activity and lean mass accretion in a neonatal pig model (El-Kadi et al. 2012, 2018). Nonetheless, continuous feeding is still the method of choice for some patients with feeding intolerance, as previous studies have shown continuous feeding to improve intestinal motor function and gastric emptying (de Ville et al. 1998), decrease energy expenditure (Grant and Denne 1991), and increase body weight (BW) gain in children with severe diarrhea (Parker et al. 1981).

On the basis of studies in cells and rodents demonstrating anabolic effects of the branchedchain AAs (BCAA), there has been recent interest in the potential for use of supplementary leucine to improve overall nitrogen retention and muscle growth. We have previously demonstrated that short-term parenteral and enteral infusion of leucine to raise circulating concentrations of leucine to fed levels enhances muscle protein synthetic activity by 
increasing the phosphorylation of mechanistic target of rapamycin complex 1 (mTORC1), and its downstream effectors, namely ribosomal protein S6 kinase 1 and eukaryotic initiation factor 4-binding protein-1 (Escobar et al. 2005; Torrazza et al. 2010; Wilson et al. 2010). To investigate the anabolic role of leucine during continuous feeding, we recently delivered parenteral pulses of leucine to neonate pigs continuously fed formula via orogastric tube over the short-term (25 h) and for a more prolonged period (Boutry et al. 2013, 2016). Compared with alanine-infused animals (i.e., control group), both short- and long-term administration of parenteral leucine pulses increased mTORC1 protein signaling in skeletal muscle and increased lean growth in those fed for 21 days, suggesting that the pro-anabolic effect of bolus feeding may be in part related to the cyclical pattern of leucine in the blood (Boutry et al. 2013, 2016). However, beyond mTORC1-related pathways, little is known about the role of leucine or its metabolites in other cellular processes that regulate muscle mass growth. To fill this gap in knowledge, our first goal was to combine transcriptome analysis of skeletal muscle with a plasma metabolomic analysis to identify if the administration of leucine pulses to neonatal pigs continuously fed formula recruits additional metabolic pathways that promote muscle anabolism.

In muscle there are several mechanisms responsible for regulating protein catabolism. Among these are the ubiquitin-proteasome and autophagy-lysosome systems (Sandri et al. 2004; Stitt et al. 2004). Insulin-stimulated protein kinase B (PKB) activity inactivates transcription factors of the Forkhead box O (FOXO) family, which in turn downregulates the expression of the E3 ubiquitin ligases muscle Atrophy F-Box/Atrogin-1 (MAFbx/atrogin-1) and muscle RING-finger protein-1 (MuRF-1), decreasing overall protein degradation via the ubiquitin-proteasome pathway (Sandri et al. 2004; Stitt et al. 2004). Conversely, activation of AMP-activated protein kinase a (AMPKa) signaling during energy stress increases the expression of ubiquitin ligases through the activation of FOXO transcription factors (Krawiec et al. 2007). Further, decreased levels of intracellular AA increases the formation of the microtubule-associated protein 1A/1B light chain 3A (LC3II), which parallels the formation of autophagosomes and leads to the activation of the autophagy-lysosomal degradation pathway (Sandri 2010). Previously, we showed that short- and long-term intermittent feeding decreased LC3II in skeletal muscle of neonatal pigs, while in the longterm, intermittent bolus feeding increased Atrogin- 1 and MuRF-1 compared with continuous feeding (El-Kadi et al. 2012, 2018). Given that short-term parenteral leucine pulses also downregulated LC3II protein abundance in neonate pigs (Boutry et al. 2013), the second goal of the present study was to investigate whether long-term administration of intermittent leucine pulses in continuously orogastric-fed pigs decreased protein catabolic pathways in muscle, and whether these changes were associated with compositional shifts in metabolomic and transcriptomic profiles.

\section{Materials and methods}

\section{Animals and experimental design}

Experiments were approved by the Institutional Animal Care and Use Committee of Baylor College of Medicine and conducted in accordance with the National Research Council's Guide for the Care and Use of Laboratory Animals (2011). The experimental design and 
tissue collection for animals used in this study were described previously (Boutry et al. 2016) and are depicted in Fig. 1a. Briefly, 23 female pigs (Sus scrofa, Yorkshire $\times$ Landrace $\times$ Hampshire $\times$ Duroc; Agriculture Headquarters, Texas Department of Criminal Justice, Huntsville, TX, USA) were weaned at 2 days of age weighing $2.0 \pm 0.2 \mathrm{~kg} \mathrm{BW}$ and housed individually in stainless steel cages in temperature-controlled rooms. Pigs were bowl-fed ad libitum a commercial milk replacement diet (Soweena ${ }^{\circledR}$ Dry Fat $7-60^{\mathrm{TM}}$; Merrick Animal Nutrition, Middleton, WI, USA) until 8 days of age, which was considered day 0 of the study (Fig. 1a). At 5 days of age piglets were anesthetized with isoflurane (PPC, Richmond Hill, Ontario, Canada) after an overnight fast, and catheters were placed surgically in the left jugular vein and carotid artery (Davis et al. 1996). The catheters were flushed with sterile heparinized saline solution (100 IU mL ${ }^{-1}$; APP, Lake Zurich, IL, USA) every $48 \mathrm{~h}$. At the same time, a gastric tube was inserted surgically for enterogastric feeding. Starting on day 0 of the study, all pigs were fed continuously $\left(240 \mathrm{~mL} \mathrm{~kg} \mathrm{BW}^{-1}\right.$, at a rate of $10 \mathrm{~mL} \mathrm{~kg} \mathrm{BW}^{-1}$ $\mathrm{h}^{-1}$ ) a liquid diet formulated to meet the nutrient requirements of 5-6 kg pigs (NRC 1998) (Tables 1,2), and randomly assigned to receive either leucine (LEU, $n=11$ ) or alanine (CON, $n=12)$ pulses into the jugular vein $\left(800 \mu \mathrm{mol} \mathrm{kg} \mathrm{BW}^{-1} \mathrm{~h}^{-1}\right)$ for $1 \mathrm{~h}$ six times per day. The leucine dose was selected based on our previous short-term study in neonatal pigs (Boutry et al. 2013), and represented 36.2\% of total leucine supplementation per day in LEU pigs. Alanine was used as the isonitrogenous control because it does not affect protein metabolism (Kim and Wu 2004). Animals were euthanized at day 21 of the study (29 days of age) using an injection of pentobarbital sodium $\left(0.4 \mathrm{~mL} \mathrm{~kg} \mathrm{BW}^{-1}\right.$; Schering-Plough, Union, NJ, USA). For metabolomic analysis, approximately $1 \mathrm{~mL}$ of blood was collected from the carotid artery into heparinized tubes $2 \mathrm{~h}$ after a leucine or alanine pulse was initiated on day 18 of the study. Blood samples were centrifuged at $2250 \mathrm{~g}$ for $15 \mathrm{~min}$ at 4 ${ }^{\circ} \mathrm{C}$, and plasma was stored at $-80{ }^{\circ} \mathrm{C}$ until further analysis. Immediately after euthanasia, approximately $5 \mathrm{~g}$ of longissimus dorsi (LD) were collected from the same site on each pig (left site, 5th rib from the top), frozen in liquid nitrogen, and stored at $-80^{\circ} \mathrm{C}$ for protein quantification and transcriptomic analysis.

\section{Metabolomic analysis}

Metabolomics were performed on plasma samples, using protein precipitation extraction with ultra-performance liquid chromatography tandem quadrupole mass spectrometry following Metabolon protocols (Metabolome, Durham, NC, USA), as previously described (Evans et al. 2014). Briefly, samples were prepared using the automated MicroLab STAR system (Hamilton, Reno, NV, USA). For recovery, standards (Evans et al. 2014) were added before the first step in the extraction process to allow confirmation of extraction efficiency. After deproteinizing the plasma samples, sample extracts reconstituted in acidic or basic LCcompatible solvents were subjected to LC/MS based on an ACQUITY ultra-performance liquid chromatography (Waters, Milford, MA, USA) and a Q-Exactive high resolution/ accurate mass spectrometer interfaced with a heated electrospray ionization (HESI-II) source and Orbitrap mass analyzer (Thermo Fisher Scientific), operating at 35,000 mass resolution. The metabolite intensities were normalized to that of internal standards added during the extraction, and to sample weight to account for small variations in starting tissue. 


\section{RNA-seq analysis}

Skeletal muscle samples from four CON and four LEU pigs, representative of the average body weight gain for each group, were used for RNA isolation and sequencing (Table 3). Total RNA was extracted using the guanidinium thiocyanate-phenol-chloroform method with TRIzol (Thermo Fisher Scientific, Waltham, MA, USA) and subsequently purified using the RNeasy Mini Kit (QIAGEN, Hilden, Germany). Purity and yield of RNA were assessed with a NanoDrop 1000 (Thermo Fisher Scientific), whereas integrity was analyzed using a 2100 BioAnalyzer (Agilent, Santa Clara, CA, USA). Only samples with RNA Integrity Number $\geq 8.5$ were sent to the Vincent J. Coates Genomics Sequences Laboratory (Berkley, CA, USA) for library preparation and sequencing. Library construction of $1 \mu \mathrm{g}$ total mRNA per sample was made using Illumina TrueSeq stranded mRNA Sample Prep Kit (Illumina, San Diego, CA, USA) according to protocol indications. RNA sequencing was performed in one sequencing lane multiplexing eight samples per lane on a HiSeq 2000 sequencer analyzer (Illumina), using a standard Illumina mRNA-seq protocol. Raw sequencing reads were trimmed by removing Illumina adapter sequences and assessed for quality using the application NGS quality control tool of the CLC Genomics Workbench software (CLC Bio, Aarhus, Denmark, EU), discarding sequences with low-quality bases and shorter than 50 bp pairs. In silico analysis of RNA-seq expression data was performed using the transcriptomics analysis tool of CLC Genomics Workbench. Sequence paired-end reads (100 bp) were mapped to the annotated porcine reference genome (https:// uswest.ensembl.org/Sus_scrofa/Info/Index). Data were normalized by calculating the 'reads per kilobase per million mapped reads' (RPKM) for each gene (Mortazavi et al. 2008). To select the expressed genes, a threshold of RPKM $\geq 0.2$ was used (Wickramasinghe et al. 2012). The up- and downregulated genes were analyzed for gene ontology (GO) and Kyoto Encyclopedia of Genes and Genomes (KEGG) pathways through the Database for Annotation, Visualization, and Integrated Discovery (DAVID) (Huang et al. 2009).

\section{Western blot analysis}

Equal amounts of protein samples were electrophoretically separated on polyacrylamide gels (PAGE; C.B.S Scientific, Del Mar, CA, USA) and transferred to PVDF membranes (Pall Corporation, Pensacola, FL, USA). The membranes were incubated overnight with primary antibodies, followed by $1 \mathrm{~h}$ incubation with secondary antibodies. Blots were developed using an enhanced chemiluminescence kit (GE Health Sciences, Buckinghamshire, UK), visualized, and analyzed using a ChemiDoc-It Imaging System ${ }^{\circledR}$ (UVP, Upland, CA, USA). For normalization, immunoblots performed with anti-phosphospecific antibodies were exposed to stripping buffer (Pierce Biotechnology, Rockford, IL, USA) and re-probed with non-phosphospecific antibodies against total protein (Cell Signaling, Danvers, MA, USA), as previously described (Davis et al. 2000). Primary antibodies used were against AMPKa $^{\text {Thr172 }}$ and total (Cell Signaling Technology), Atrogin-1 (ECM Biosciences, Versailles, KY, USA), MuRF-1 (R\&D Systems, Minneapolis, MN, USA), FOXO1 ${ }^{\mathrm{Ser} 256}$ and total (Cell Signaling Technology), LC3I/II (Cell Signaling Technology), FOXO3a ${ }^{\mathrm{Ser} 253}$ and total (Cell Signaling Technology), sirtuin 1 (SIRT-1; Cell Signaling Technology), protein kinase B ( $\mathrm{PKB}^{\mathrm{Ser} 473}$, Thr308 and total; Cell Signaling Technology), serine/threonine kinase 4

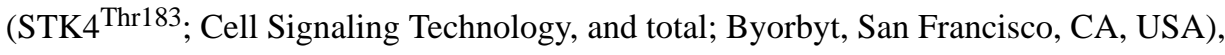


beta-actin (Bethyl Laboratories; Montgomery, TX, USA), and NUAK family SNF1-like kinase 2 (NUAK2; Byorbyt).

\section{Statistical analyses}

Western blot data were analyzed by a one-way ANCOVA using a linear model in SAS (v. 9.2; SAS Institute Inc., Cary, NC, USA), with treatment (CON, LEU) as a fixed effect, and initial BW as the covariate. The normality of the residuals and presence of outliers were assessed, and non-normally distributed parameters were power transformed by a parameter $\varphi$ whose optimal value was estimated using the maximum likelihood method (Piepho 2009). Data are presented as means \pm SD. $P$ values were calculated using Student's $t$ tests, and significant effects were considered at $P \leq 0.05$.

Metabolomics data were imported into the Primer-E software (v.7; Primer-E Ltd., Plymouth, UK), log-transformed into a normal distribution approximation, and analyzed with a Euclidean distance matrix. A non-parametric permutational analysis of variance (PERMANOVA; Primer-E Ltd) with treatment as fixed effects, and BW as the covariate, was used for testing the null hypothesis of no difference between groups under a reduced model, 9999 permutations, and type III sum of squares. Metabolomics data were further assessed by Principal Component Analysis (PC) to visualize group discrimination in a 2dimensional scores plot. PC analyses were conducted in the R Statistical Language using the clustvis online tool (Huang et al. 2009). The identification of metabolites differentially expressed between groups was performed by a one-way ANOVA with the same fixed effects and covariables described above. $P$ values were calculated using Student's $t$ tests. Data are presented as fold change (LEU/CON). Significant effects were considered at $P \leq 0.05$ and trends at $P \leq 0.1$.

RNA-seq data were transformed from negative binomial distribution to normal distribution. The identification of metabolites differentially expressed between groups (LEU/CON) was performed by a one-way ANOVA with the same fixed effects and covariables described above. $P$ values were calculated using Student's $t$ tests. Data are presented as fold change. Significant effects were considered at $P \leq 0.05$ and trends at $P \leq 0.1$.

Significant associations between the skeletal muscle genes and metabolites altered by leucine pulses was assessed by Spearman's correlations in $R$ and then visualized with a correlation network. Before correlation analysis, missing metabolomics data were imputed using the $k$-nearest neighbor imputation method (Troyanskaya et al. 2001; Hastie et al. 2019) and then log-transformed. Spearman's correlations were assessed for all genes and metabolites previously shown to have statistically significant group differences. All pairwise correlations were determined with the rcorr() function in the Hmisc package (Harrell and Dupont 2019), followed by adjustment for multiple comparisons using the false discovery rate (FDR) procedure (Benjamini and Hochberg 1995). Gene-metabolite pairwise correlations were extracted, filtered at FDR $\leq 0.1(n=88)$, then visualized in a correlation network using Cytoscape v3.7.1. 


\section{Results}

\section{Effect of leucine vs. alanine pulses in plasma metabolome}

To investigate the systemic mechanisms leading to increased protein accretion in leucinesupplemented pigs, we performed a comprehensive analysis of metabolites in plasma. A total of 483 metabolites were detected, of which 37 differed between CON and LEU groups. Multivariate analysis showed a separation of samples in two differentiated clusters in the PC chart (Fig. 1b). A complete list of the metabolites analyzed is included in Online Resource 1. Compared with CON, LEU increased leucine and metabolites related to leucine catabolism, including 4-methyl-2-oxopentanoate, $\beta$-hydroxyisovalerate, $\beta$-hydroxyisovalerylcarnitine, and 3-methylglutaconate $(P \leq 0.05$; Fig. 1c). Conversely, leucine pulses decreased multiple plasma metabolites related to AA metabolism, including alanine $(P \leq 0.05), N$-acetylserine ( $P \leq 0.05)$, 4-guanidinobutanoate $(P \leq 0.05)$, 4-imidazoleacetate $(P \leq 0.05), N 6, N 6, N 6$ trimethyllysine $(P \leq 0.05), N$-acetyl methionine sulfoxide $(P \leq 0.01)$, cysteine s-sulfate $(P \leq$ $0.05)$, acisoga $(P \leq 0.05)$, as well as valine and isoleucine-related metabolite glycylisoleucine $(P \leq 0.05)$. LEU also decreased $N$ l-methyl-2-pyridone-5-carboxamide and quinolinate $(P \leq 0.05)$, involved in nicotinamide metabolism, carboxylic acid methylenesuccinate $(P \leq 0.05)$, fatty acid-related metabolites hexanoylglycine $(P \leq 0.05), 1$ dihomo-linoleoylglycerol $(P \leq 0.05)$, and 3-hydroxysebacate $(P \leq 0.05)$, and multiple glycerol conjugates, including 1-myristoyl, 1- and 2-linoleoyl ( $P \leq 0.001), 1$-palmitoleoyl ( $P$ $\leq 0.01)$. 1- and 2-palmitoyl $(P \leq 0.01$ and $P \leq 0.05), 1$ - and 2-oleoyl $(P \leq 0.05)$, 1-dihomolinolenyl ( $P \leq 0.05), 1$-arachidonyl $(P \leq 0.05), 1$ and 2-stearoyl $(P \leq 0.05)$, and docosatrienoate $(P \leq 0.05)$. Secondary bile acids 6-oxolithocholate and ursodeoxycholate $(P$ $\leq 0.05)$, and xenobiotic 2-pyrrolidinone ( $P \leq 0.05)$, were also lower in LEU compared with CON.

\section{Transcriptomic response in skeletal muscle}

We performed transcriptome profiling on LD samples isolated from representative LEU ( $n=$ 4) and CON $(n=4)$ pigs. On average, Ilumina MiSeq sequencing generated 46,072,339 paired-end reads for each sample that, following analysis with CLC Genomic Work Bench, resulted in 22,861 genes (62.68\% of reads) mapped to the porcine reference genome (Table 3 ). Figure 2 shows that the LD samples from LEU pigs, and corresponding CON animals, were separated into two different clusters by hierarchical analysis, using the normalized mean expression of the whole transcriptome across the eight libraries. When comparing the skeletal muscle gene signature of the LEU pigs to the CON, we found 465 differentially expressed genes (DEGs; $P \leq 0.05$ ), with 144 transcripts upregulated in the LEU group, and 321 transcripts downregulated relative to the CON group $(P \leq 0.05)$. A complete list of all DEGs is included in Online Resource 2. The heat map in Fig. 3a, and PC in Fig. 3b show very similar changes in the expression of the 465 DEGs within treatments, but a clear separation between CON and LEU pigs.

The DAVID gene functional classification tool was used to identify groups of transcripts among DEGs sharing common KEGG terms (Fig. 3c). Compared to CON, LEU pigs showed downregulated transcripts enriched in "spliceosome" (ssc03040, $P \leq 0.028$, fold

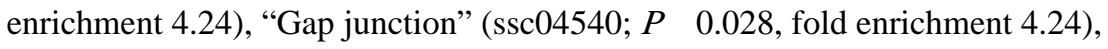


"endocytosis" (ssc04144; $P \leq 0.054$, fold enrichment 7.21), "extracellular matrix-receptor interaction" ( $\operatorname{ssc} 04512 ; P \leq 0.036$, fold enrichment 5.40), and "DNA replication" (ssc03030; $P \leq 0.039$, fold enrichment 9.38). To obtain more detailed gene expression patterns, DEGs were associated with GO biological process categorization using the software DAVID (Table 4). The transcripts downregulated in the LEU group were significantly enriched in biological processes, such as "replication fork processing" (GO:0031297; $P \leq 0.01$, fold enrichment 20.9 ) and "double-strandbreak repair via homologous recombination" (GO:0000724; $P \leq$ 0.05 , fold enrichment 8.36). In the cellular component category, genes associated with "nucleoplasm" (GO:0005654; $P \leq 0.001$, fold enrichment 2.07), "minichromosome maintenance protein complex" (GO:00042555; $P \leq 0.01$, fold enrichment 47.5), "nuclear chromatin" (GO:0000790; $P \leq 0.01$, fold enrichment 5.18), "microtubule cytoskeleton" (GO:0015630; $P \leq 0.01$, fold enrichment 6.71) and "nucleolus" (GO:0005730; $P \leq 0.05$, fold enrichment 2.27) were identified as the most significantly down-regulated terms in LEU, whereas among molecular functions, the terms "DNA helicase activity" (GO:0003678; $P \leq$ 0.001, fold enrichment 63.3), "RNA binding" (GO:0003723; $P \leq 0.05$, fold enrichment 3.1), "zinc ion binding" (GO:0008270; $P \leq 0.05$, fold enrichment 1.70), and "mRNA binding" (GO:0003729; $P \leq 0.05$, fold enrichment 4.69) decreased in LEU compared with CON. The transcripts upregulated for LEU as compared with CON pigs were significantly enriched in "extracellular exosome" (GO:0070062; $P \leq 0.05$, fold enrichment 1.69) and "single-stranded DNA binding" (GO:0003697; $P \leq 0.05$, fold enrichment 10.6).

Applying the most stringent parameters (RPKM $P \leq 0.01+$ fold change $>2$ ), 18 DEGs were identified, of which 12 were mapped to the porcine reference genome (Fig. 3d). Genes differentially regulated in the LEU compared with the CON group were associated with myocyte survival and maintenance of muscle (NUAK2), signaling and cellular processes (GPR37L1, CHRNA3, REPS2), neural formation and connections (ANKRD6), muscle atrophy ( $S T K 4)$, cell division (CCNJL, ZBTB32, CDCA2), and mitochondrial activity and electron transfer ( $A L D H 4 A 1, T M E M 117)$.

\section{Correlation of transcriptome and metabolites}

The correlation analysis between RNA-seq data and plasma metabolome revealed that changes in leucine-derived metabolites, primary bile acids, lipids, tricarboxylic acid intermediates, and xenobiotics were associated with specific changes of the muscle transcriptome ( $P \leq 0.01$; Fig. 4). Classification in categories of genes significantly correlated with metabolites was performed manually based on information obtained in the UniProt database (UniProt Consortium 2019). Significant associations were identified between leucine-derived metabolites $\beta$-hydroxyisovalerate, $\beta$-hydroxyisovalerylcarnitine, and 3methylglutaconate, which increased in the LEU compared with the CON pigs, and expression of LD genes involved in protein degradation (negative: $S N X 32$; positive: TRIM62 and $O P T N$ ), transcription and translation (negative: PARP8, OFD1, SF3B4; positive: $U B L 4 A$ ), cell cycle regulation and DNA replication (negative: $A S F 1 B$; positive: HAUS1, ROMO1, MFAP3L, and $A N K H$ ), muscle maintenance and development (positive: $P O P D C 3$ ), apoptosis (negative: $S E R B P 1$; positive: TMEM117), extracellular matrix (positive: $C D 9$ ), and $\mathrm{G}$ protein receptors (negative: $G R M 7$; positive, $R G S 3$ ). Plasma levels of glycocholate correlated with genes involved in cell cycle regulation and DNA replication 
(negative: $K L H D C 3, R N F 17$; positive: $Z N F 652$ and $M F A P 3 L$ ), transcription and translation (negative: PARP8, OFD1, and SF3B4) and G protein receptors (positive, GNRHR2).

Among metabolites decreased by leucine pulses, 3-hydroxy-sebacate was associated with genes associated with muscle maintenance and development (positive: STK4), cell cycle regulation and DNA replication (positive: ZNF207 and INO80), extracellular matrix (positive: $V N N 2$ and $L P X N$ ), apoptosis (positive: $B C L-X L$ and $E R L E C 1$ ), and energy (positive: $H M G C L L 1$ and $P D E 1 O A$ ). Itaconate was also correlated with genes involved in autophagy (negative, SNX32), extracellular matrix (positive: $M M P 15$ ), G protein receptors (positive, $R G S 3$ ), and transcription and translation (negative: ESF1), whereas 2pyrrolidinone was associated with genes involved in endocytosis (negative: AP4S1; positive: $O P H N 1$ ), transcription and translation (positive: $T B X 21$ ), G protein receptors (negative, TAS2R4O), DNA replication (positive: DSCC1, ZNF385D, and ABLIM3), and extracellular matrix (positive: $F N 1$ ).

\section{Protein signaling and abundance in skeletal muscle}

Western Blot data pertain to the 23 pigs used in the study. Previously, we demonstrated that short-term pulsatile administration of leucine in continuously fed neonate pigs increased mTORC1 signaling and suppressed autophagy (Boutry et al. 2013), which in turn positively affects protein accretion in skeletal muscle (Boutry et al. 2016). To investigate whether longterm administration of intermittent leucine pulses in continuously orogastric-fed pigs was associated with the downregulation of protein degradation pathways in LD, we assessed the expression of macroautophagy and ubiquitin-proteasome-related proteins and regulatory kinases. In addition, we investigated the protein expression of novel kinases related to the maintenance of muscle fiber and autophagy previously identified in the transcriptomic analysis. We found a significant lower LC3II/LC3 ratio in the LEU compared with the CON pigs $(P \leq 0.01)$, whereas LC3 total abundance did not change between groups (Fig. 5a). No differences between the LEU and CON groups were observed in SIRT1, total and phosphorylated PKB and AMPK, phosphorylated FOXO1 and FOXO3, and total MURF-1 and Atrogin-1 (Fig. 5b, c). We also did not find differences between the LEU and CON pigs for NUAK2, whereas both total and phosphorylated STK4 were lower in the muscle of LEU compared to CON pigs ( $P \leq 0.05$; Fig. 5 d).

\section{Discussion}

\section{Intermittent leucine pulses in continuously orogastric-fed pigs differentially affect peptides and essential AA levels in plasma}

Orogastric tubes are commonly used in premature and term infants who cannot meet their nutrient requirements for optimal growth due to their inability to be breast- or bottle-fed (Hill et al. 1995; Dziechciarz et al. 2007; Braegger et al. 2010). However, the impact of enterogastric feeding modality (i.e., continuous vs. intermittent) in the molecular pathways influencing muscle protein synthetic activity and gain during the neonatal period are incompletely known. Previously we demonstrated that the stimulatory effect of intermittent bolus feeding on skeletal muscle growth is partially related to the pulsatile pattern of AA and insulin in the blood (El-Kadi et al. 2012, 2018). These effects were associated with the 
activation of the mTORC1 signaling pathway and stimulation of protein synthetic rates and could be largely reproduced by the parenteral supplementation of leucine administered in a cyclical pattern to neonatal pigs continuously fed by orogastric tube (Boutry et al. 2013, 2016). To understand further the molecular mechanisms by which long-term leucine pulses during continuous feeding increase muscle protein accretion, we now examined the metabolomic profile in plasma samples collected in our previous study (Boutry et al. 2016). Compared with the control animals pulsed with alanine, plasma levels of leucine were higher in the leucine supplemented pigs, along with multiple leucine-containing dipeptides such as lysyl leucine and glutamine-leucine. In contrast, analytes related to indispensable AA metabolism were decreased, suggesting an increase in the incorporation of indispensable AA into proteins, and thereby depleting their pool. As expected, the infusion of leucine led to an increase in the a-ketoacid 4-methyl-2-oxopentanoate, as well as further downstream metabolites associated with leucine catabolism, including $\beta$-hydroxyisovalerylcarnitine, $\beta$ hydroxyisovalerate, and 3-methylglutaconate, indicative of increased leucine catabolism. Conversely, valine and isoleucine-related metabolites such as $\mathrm{N}$-acetylisoleucine and glycylisoleucine decreased in response to leucine pulses, while the corresponding $a-$ ketoacids 3-methyl-2-oxobutyrate and 3-methyl-2-oxovalerate increased, though not significantly (Online Resource 1), in LEU compared with CON piglets. These results are consistent with our earlier findings that the post-prandial levels of valine and isoleucine are impacted by both parenteral (Boutry et al. 2013, 2016) and enteral leucine supplementation (Manjarín et al., 2016, 2018), likely due to the leucine-induced upregulation of the activity of the branched chain (BC)-keto acid dehydrogenase enzymatic complex, which results in the catabolism of all BCAAs (Tannous et al. 1963; Harper et al. 1970).

\section{Lean mass gain in LEU pigs was associated with a decrease of lipid metabolites}

In addition to the increase in BW and lean mass gain, the delivery of intermittent parenteral leucine pulses decreased body fat by almost $50 \%$ compared with CON pigs supplemented with alanine (Boutry et al. 2016). Similar results have been observed in human and animal studies, in which AA supplemented diets aimed at increasing the plasma concentration of leucine or other BCAAs led to consistent improvement in body composition (Børsheim et al. 2008; Chen Scarabelli et al. 2008). Increased BCAA catabolism has been related to increased fatty acid oxidation via the tricarboxylic acid cycle and glycerol neogenesis resulting in a lean metabolic phenotype (Kainulainen et al. 2013). Interestingly, one of the most consistent and strongest leucine-associated effects found in this study was the pronounced decrease in nearly all monoacylglycerols, as well as carboxylic acid methylene succinate, and several fatty acid-related metabolites, suggesting an increase in fatty acid oxidation in pigs supplemented with leucine.

\section{Skeletal muscle transcriptome in continuously orogastric-fed pigs was regulated by intermittent leucine pulses}

To our knowledge, this is the first time that RNA-seq has been used to elucidate changes in the skeletal muscle transcriptome in response to intermittent leucine pulses. While we have previously demonstrated that leucine is a key factor regulating muscle mass gain during the neonatal period through protein changes in the mTORC1 signaling pathway (Escobar et al. 2005; Torrazza et al. 2010; Wilson et al. 2010), the present results also show an impact of 
leucine at the skeletal muscle transcriptional level. Out of 22,861 detected genes, 465 were affected in skeletal muscle of leucine supplemented pigs, with the expression of almost $70 \%$ of them being downregulated as compared with controls. The analysis of KEGG pathways and GO biological process categorization revealed a consistent decrease in terms related to "spliceosome" in LEU compared with CON pigs. The spliceosome is responsible for posttranscriptional processing of pre-mRNA into mature mRNA (Pandya-Jones 2011). It is possible that a decrease in splicing underlies a regulatory mechanism for gene expression in skeletal muscle, as many protein-coding genes possess only a few or no introns that can be spliced even in the presence of few functional spliceosomes, whereas the expression of genes with a high number of introns becomes downregulated (Jeffares et al. 2008; Singh and Padgett 2009). Alternatively, the downregulation of spliceosomal genes in LEU pigs may not lead to a decrease in splicing efficiency in skeletal muscle, given that splicing is regulated by a complex network of activating and inhibiting splicing factors (Anufrieva et al. 2018). In this regard, removal of spliceosomal proteins increased histone H2AX phosphorylation and activated DNA repair in cancer cell lines, increasing genome stability (Paulsen et al. 2009).

Terms associated with "cell cycle" and "DNA replication" were also decreased in LEU compared with CON in both the KEGG pathway and GO categorization analyses. Interestingly, a parallel downregulation in the expression of spliceosomal and cell cyclerelated genes has also been demonstrated in cancer cells in response to various stresses to promote their survival (Anufrieva et al. 2018), as well as in mice and human cell lines (Kittler et al. 2004; Kleinridders et al. 2009), suggesting a connection between pre-mRNA splicing and cell cycle progression. Additional terms downregulated in LEU pigs included "GAP junction" and "ECM-interaction" in KEGG, as well as "MCM complex" and "proteinaceous extracellular matrix" in GO analysis. Differential expression of genes in these pathways requires further investigation, as extracellular membrane matrix components such as integrins, are associated with PKB-mTORC1 signaling (Goc et al. 2011; Bui et al. 2019), and therefore may be involved in leucine-induced protein synthesis. Of note, only three DEGs were mapped to "PI3K-Akt signaling pathway" in KEGG (ssc04151), and none

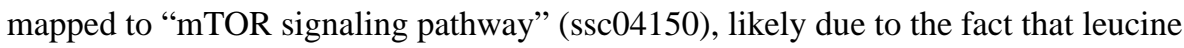
activates mTOR1 signaling by increasing the phosphorylation of kinases, such as AKT and S6K1/2, rather than by upregulating the transcription/translation of genes.

\section{Leucine pulses decrease markers of autophagy and endocytosis in continuously orogastric-fed pigs}

Autophagy can be activated by starvation, exercise, and a variety of stress signals, and promotes muscle protein degradation by increasing the breakdown of damaged proteins and organelles (Sandri 2010). Consistent with our previous studies (Boutry et al. 2013, 2016; ElKadi et al. 2018), we observed a significant decrease in LC3II: LC3 ratio in response to leucine pulses, which reflects a decrease in the number of autophagosomes and autophagyrelated structures (Yoshii and Mizushima 2017). Interestingly, "endocytosis" was also significantly downregulated in skeletal muscle of LEU compared with CON pigs. The endosome system functions in importing nutrients and macromolecules into the cell from outside and acts in close relationship with autophagy to provide AAs and other basic components for use by the cell (Tooze et al. 2014). In the endocytic pathway, vesicles 
formed by clathrin-dependent or -independent mechanisms from the plasma membrane fuse and deliver their membrane and contents to endosomes, which increase in size before being targeted to lysosomes for the enzymatic digestion of enclosed material (Tooze et al. 2014). Additionally, endosomes are capable of fusing with autophagosomes to form amphisomes which go on to fuse with the lysosome and are also degraded (Hyttinen et al. 2013; Tooze et al. 2014). Although speculative, leucine-induced downregulation of genes involved in endocytosis may imply that leucine pulses not only decrease protein degradation in neonatal pigs by inhibiting the formation of autophagosomes but also by downregulating the endosome-autophagy pathway.

In addition to autophagy markers, a kinase that controls muscle atrophy, STK4 (Wei et al. 2013), was also downregulated at both gene and protein expression levels in muscle of the leucine supplemented pigs. The mammalian STK family is expressed in multiple organs, including skeletal muscle, where it promotes the expression of $L C 3$ and Atrogin-1 through activation of FOXO transcription factors (Wei et al. 2013). Interestingly, protein expression of FOXOs, MuRF-1, and Atrogin-1 did not differ between LEU and CON pigs in our current and previous studies (Boutry et al. 2013), whereas both E3 ligases increased in bolus-fed pigs (El-Kadi et al. 2012, 2018). Taken together, it is possible that leucine pulses, through STK4 downregulation, inhibit the activation of the ubiquitin-proteasome pathway in neonatal muscle. Conversely, the expression of AMPK-related kinase NUAK2, which has an antiapoptotic role in myocyte survival and maintenance of muscle mass (Lessard et al. 2016), was increased at the transcript level in LEU compared with CON pigs. Nonetheless, we did not observe differences in NUAK2 protein abundance between groups, which might be due to post-transcriptional regulation, or changes in protein degradation or turnover rates.

\section{Correlation analysis revealed leucine-derived metabolites associated with expression of genes involved in protein degradation, transcription, and translation in neonatal muscle}

We have previously shown that leucine-derived metabolites a-ketoisocaproic acid (KIC) and $\beta$-hydroxy- $\beta$-methylbutyrate can activate translation initiation factors and protein synthesis in skeletal muscle of neonatal pigs (Escobar et al. 2010; Wheatley et al. 2014; Kao et al. 2016; Suryawan et al. 2020). We now extend these findings to show that other metabolites derived from leucine catabolism, including $\beta$-hydroxyisovalerate, $\beta$ hydroxyisovalerylcarnitine, and 3-methylglutaconate, are strongly correlated with the expression of LD genes associated with protein degradation, transcription, and translation, DNA replication, and muscle maintenance and development. The first step in leucine catabolism is the reversible transamination of leucine to KIC, catalyzed by branched-chain aminotransferase isoenzymes in muscle tissue, followed by the conversion of KIC to $\beta$ hydroxy- $\beta$-methyl butyrate by $\alpha$-ketoisocaproic dioxygenase, or to isovaleryl-CoA (a precursor of $\beta$-hydroxyisovalerate and $\beta$-hydroxyisovalerylcarnitine) by KIC dehydrogenase in the liver (Kohlmeier 2015). Of particular significance for our study is the association of $\beta$ hydroxyisovalerylcarnitine and $\beta$-hydroxyisovalerate with $O P T N$ and $S N X 32$, involved in the regulation of macroautophagy, as well as TRIM62, that encodes an E3 ubiquitin ligase. Interestingly, enteral or parenteral supplementation of $\beta$-hydroxy- $\beta$-methylbutyrate to neonatal pigs did not affect the abundance of ubiquitin ligases or LC3II/LC3 ratio in muscle samples (Wheatley et al. 2014; Kao et al. 2016). These results suggest that metabolites from 
leucine catabolism differ in their mechanisms to enhance muscle protein accretion, with those derived from the KIC dehydrogenase pathway being able to regulate both protein synthesis and degradation signaling in neonatal pigs.

In conclusion, results from our studies support the notion that the administration of intermittent leucine pulses in continuously orogastric-fed pigs enhances muscle mass gain not only by increasing protein synthetic activity but also by reducing the expression of protein catabolic pathways, as indicated by changes in the transcriptome and metabolome. The novel association between metabolites derived from leucine catabolism and markers of the ubiquitin-proteasome and autophagy-lysosome systems needs to be further studied, as it may help to develop better therapeutic approaches for improving muscle growth in at-risk human infants.

\section{Supplementary Material}

Refer to Web version on PubMed Central for supplementary material.

\section{Funding}

The work was supported by National Institute of Arthritis and Musculoskeletal and Skin Diseases Grants AR-044474 (Davis) and AR-46308 (Fiorotto), National Institute of Child Health and Human Development HD-072891 (Davis), HD085573 (Davis), H***D099080 (Davis and Fiorotto), United States Department of Agriculture National Institute of Food and Agriculture Grant 2013-67015-20438 (Davis), California State University Agricultural Research Institute grant 58982 (Manjarin and Maj), and by the USDA/ARS under Cooperative Agreement No. 6250-510000-055 (Davis). This work is a publication of the USDA, Agricultural Research Service (USDA/ARS) Children's Nutrition Research Center, Department of Pediatrics, Baylor College of Medicine, Houston, TX, USA. The contents of this publication do not necessarily reflect the views or politics of the USDA, nor does the mention of trade names, commercial products, or organizations imply endorsement by the U.S. Government.

\section{Abbreviations}

AA

ABLIM3

ALDH4A1

AMPKa

ANKH

ANKRD6

AP4S1

ASF1B

AU

BCAA

BCL-XL
Amino acids

Actin binding LIM protein family member 3

Aldehyde dehydrogenase 4 family member A1

AMP-activated protein kinase a

ANKH inorganic pyrophosphate transport regulator

Ankyrin repeat domain 6

Adaptor related protein complex 4 sigma 1 subunit

Anti-silencing function 1B histone chaperone

Arbitrary units

Branched-chain amino acids

B-cell lymphoma-extra large 


\begin{tabular}{|c|c|}
\hline BW & Body weight \\
\hline CCNJL & Cyclin J like \\
\hline CD9 & CD9 molecule \\
\hline CDCA2 & Cell division cycle associated 2 \\
\hline CHRNA3 & Cholinergic receptor nicotinic alpha 3 subunit \\
\hline $\mathrm{CON}$ & Control group \\
\hline DAVID & Database for Annotation, Visualization, and Integrated Discovery \\
\hline DEGs & Differentially expressed genes \\
\hline DSCC1 & DNA replication and sister chromatid cohesion 1 \\
\hline EAA & Essential amino acids \\
\hline ERLEC1 & Endoplasmic reticulum lectin 1 \\
\hline ESF1 & ESF1 nucleolar pre-rRNA processing protein, homolog \\
\hline FN1 & Fibronectin 1 \\
\hline FOXO & Forkhead box protein $\mathrm{O}$ \\
\hline GNRHR2 & Gonadotropin-releasing hormone II receptor \\
\hline GO & Gene ontology \\
\hline GPR37L1 & G protein-coupled receptor 37 like 1 \\
\hline GRM7 & Glutamate metabotropic receptor 7 \\
\hline HAUS1 & HAUS augmin like complex subunit 1 \\
\hline HMGCLL1 & 3-Hydroxymethyl-3-methylglutaryl-CoA lyase like 1 \\
\hline INO80 & Chromatin-remodeling ATPase INO80 \\
\hline KEGG & Kyoto encyclopedia of genes and genomes \\
\hline KIC & a-Ketoisocaproic acid \\
\hline KLHDC3 & Kelch domain containing 3 \\
\hline LC3I/II & Microtubule-associated protein $1 \mathrm{~A} / 1 \mathrm{~B}$ light chain $3 \mathrm{~A}$ \\
\hline LD & Longissimus dorsi \\
\hline LEU & Leucine supplemented group \\
\hline LPXN & Leupaxin \\
\hline MAFbx & Muscle Atrophy F-Box/Atrogin-1 \\
\hline
\end{tabular}




\begin{tabular}{|c|c|}
\hline MFAP3L & Microfibrillar associated protein 3 like \\
\hline MMP15 & Matrix metallopeptidase 15 \\
\hline mTORC1 & Mammalian target of rapamycin complex 1 \\
\hline MuRF-1 & Muscle RING-finger protein-1 \\
\hline NUAK2 & NUAK family SNF1-like kinase 2 \\
\hline OFD1 & Oral-facial-digital syndrome 1 protein \\
\hline OPHN1 & Oligophrenin 1 \\
\hline OPTN & Optineurin \\
\hline $\mathbf{P} / \mathbf{T}$ & Phosphorylated/total protein \\
\hline PARP8 & Protein mono-ADP-ribosyltransferase \\
\hline PC & Principal component analysis \\
\hline PDE10A & Phosphodiesterase 10A \\
\hline PKB/Akt & Protein kinase B \\
\hline POPDC3 & Popeye domain containing 3 \\
\hline REPS2 & RalBP1-associated Eps domain-containing 2 \\
\hline RGS3 & Regulator of G-protein signaling 3 \\
\hline RNA-seq & High-throughput RNA sequencing \\
\hline RNF17 & Ring finger protein 17 \\
\hline ROMO1 & Reactive oxygen species modulator 1 \\
\hline RPKM & Reads per kilobase per million mapped reads \\
\hline SERBP1 & Plasminogen activator inhibitor 1 RNA-binding protein \\
\hline SF3B4 & Splicing factor $3 \mathrm{~b}$ subunit 4 \\
\hline SIRT-1 & Sirtuin 1 \\
\hline SNX32 & Sorting nexin 32 \\
\hline STK4 & Serine/threonine kinase 4 \\
\hline TAS2R40 & Taste 2 receptor member 40 \\
\hline TBX21 & T-box 21 \\
\hline TMEM117 & Transmembrane protein 117 \\
\hline UBL4A & Ubiquitin like $4 \mathrm{~A}$ \\
\hline
\end{tabular}


VNN2 Vanin 2

ZBTB32 Zinc finger and BTB domain containing 32

ZNF207 Zinc finger protein 207

ZNF385D Zinc finger protein 385D

ZNF652 Zinc finger protein 652

\section{References}

Anufrieva KS, Shender VO, Arapidi GP, Pavlyukov MS, Shakhparonov MI, Shnaider PV, Butenko I, Lagarkova MA, Govorun VM (2018) Therapy-induced stress response is associated with downregulation of pre-mRNA splicing in cancer cells. Genome Med 10(1):49 [PubMed: 29950180]

Aynsley-Green A, Adrian TE, Bloom SR (1982) Feeding and the development of enteroinsular hormone secretion in the preterm infant: effects of continuous gastric infusions of human milk compared with intermittent boluses. Acta Paediatr Scand 71(3):379-383 [PubMed: 6814175]

Benjamini Y, Hochberg Y (1995) Controlling the false discovery rate: a practical and powerful approach to multiple testing. J R Stat Soc 57:289-300

Børsheim E, Bui QU, Tissier S, Kobayashi H, Ferrando AA, Wolfe RR (2008) Effect of amino acid supplementation on muscle mass, strength and physical function in elderly. Clin Nutr 27(2):189195 [PubMed: 18294740]

Boutry C, El-Kadi SW, Suryawan A, Wheatley SM, Orellana RA, Kimball SR, Nguyen HV, Davis TA (2013) Leucine pulses enhance skeletal muscle protein synthesis during continuous feeding in neonatal pigs. Am J Physiol Endocrinol Metab 305(5):E620-E631 [PubMed: 23839523]

Boutry C, El-Kadi SW, Suryawan A, Steinhoff-Wagner J, Stoll B, Orellana RA, Nguyen HV, Kimball SR, Fiorotto ML, Davis TA (2016) Pulsatile delivery of a leucine supplement during long-term continuous enteral feeding enhances lean growth in term neonatal pigs. Am J Physiol Endocrinol Metab 310(8):E699-E713 [PubMed: 26884386]

Braegger C, Decsi T, Dias JA, Hartman C, Kolacek S, Koletzko B, Koletzko S, Mihatsch W, Moreno L, Puntis J, Shamir R, Szajewska H, Turck D, van Goudoever J, ESPGHAN Committee on Nutrition (2010) Practical approach to pediatric enteral nutrition: a comment by the ESPGHAN committee on nutrition. J Pediatr Gastroenterol Nutr 51(1):110-122 [PubMed: 20453670]

Bui T, Rennhack J, Mok S, Ling C, Perez M, Roccamo J, Andrechek ER, Moraes C, Muller WJ (2019) Functional redundancy between $\beta 1$ and $\beta 3$ integrin in activating the IR/Akt/mTORC1 signaling axis to promote ErbB2-driven breast cancer. Cell Rep 29(3):589-602.e6 [PubMed: 31618629]

Chen Scarabelli C, McCauley RB, Yuan Z, Di Rezze J, Patel D, Putt J, Raddino R, Allebban Z, Abboud J, Scarabelli GM, Chilukuri K, Gardin J, Saravolatz L, Faggian G, Mazzucco A, Scarabelli TM (2008) Oral administration of amino acidic supplements improves protein and energy profiles in skeletal muscle of aged rats: elongation of functional performance and acceleration of mitochondrial recovery in adenosine triphosphate after exhaustive exertion. Am J Cardiol 101(11A):42E-48E

da Huang W, Sherman BT, Lempicki RA (2009) Systematic and integrative analysis of large gene lists using DAVID bioinformatics resources. Nat Protoc 4:44-57 [PubMed: 19131956]

Davis TA, Burrin DG, Fiorotto ML, Nguyen HV (1996) Protein synthesis in skeletal muscle and jejunum is more responsive to feeding in 7-than in 26-day-old pigs. Am J Physiol 270(5 Pt 1):E802-E809 [PubMed: 8967468]

Davis TA, Nguyen HV, Suryawan A, Bush JA, Jefferson LS, Kimball SR (2000) Developmental changes in the feeding-induced stimulation of translation initiation in muscle of neonatal pigs. Am J Physiol Endocrinol Metab 279:E1226-E1234 [PubMed: 11093908]

de Ville K, Knapp E, Al-Tawil Y, Berseth CL (1998) Slow infusion feedings enhance duodenal motor responses and gastric emptying in preterm infants. Am J Clin Nutr 68:103-108 [PubMed: 9665103] 
Dziechciarz P, Horvath A, Shamir R, Szajewska H (2007) Meta-analysis: enteral nutrition in active Crohn's disease in children. Aliment Pharmacol Ther 26(6):795-806 [PubMed: 17767463]

El-Kadi SW, Suryawan A, Gazzaneo MC, Srivastava N, Orellana RA, Nguyen HV, Lobley GE, Davis TA (2012) Anabolic signaling and protein deposition are enhanced by intermittent compared with continuous feeding in skeletal muscle of neonates. Am J Physiol Endocrinol Metab 302(6):E674E686 [PubMed: 22215651]

El-Kadi SW, Boutry C, Suryawan A, Gazzaneo MC, Orellana RA, Srivastava N, Nguyen HV, Kimball SR, Fiorotto ML, Davis TA (2018) Intermittent bolus feeding promotes greater lean growth than continuous feeding in a neonatal piglet model. Am J Clin Nutr 108(4):830-841 [PubMed: 30239549]

Escobar J, Frank JW, Suryawan A, Nguyen HV, Kimball SR, Jefferson LS, Davis TA (2005) Physiological rise in plasma leucine stimulates muscle protein synthesis in neonatal pigs by enhancing translation initiation factor activation. Am J Physiol Endocrinol Metab 288:E914-E921 [PubMed: 15644455]

Escobar J, Frank JW, Suryawan A, Nguyen HV, Van Horn CG, Hutson SM, Davis TA (2010) Leucine and alpha-ketoisocaproic acid, but not norleucine, stimulate skeletal muscle protein synthesis in neonatal pigs. J Nutr 140(8):1418-1424 [PubMed: 20534881]

Evans AM, Bridgewater BR, Liu Q, Mitchell MW, Robinson RJ, Dai H, Stewart SJ, DeHaven CD, Miller LAD (2014) High resolution mass spectrometry improves data quantity and quality as compared to unit mass resolution mass spectrometry in high-throughput profiling metabolomics. Metabolomics 4:132

Goc A, Choudhary M, Byzova TV, Somanath PR (2011) TGF $\beta$ - and bleomycin-induced extracellular matrix synthesis is mediated through Akt and mammalian target of rapamycin (mTOR). J Cell Physiol 226(11):3004-3013 [PubMed: 21302298]

Grant J, Denne SC (1991) Effect of intermittent versus continuous enteral feeding on energy expenditure in premature infants. J Pediatr 118:928-932 [PubMed: 1904091]

Harper AE, Benevenga NJ, Wohlhueter RM (1970) Effects of ingestion disproportionate amounts of amino acids. Physiol Rev 50:428-558 [PubMed: 4912906]

Harrell FE, Dupont C (2019) Hmisc: Harrell Miscellaneous. R package version 4.2-0. https:// CRAN.R-project.org/package=Hmisc. Accessed 13 Jan 2020

Hastie T, Tibshirani R, Narasimhan B, Chu G (2019) Imputation for microarray data. R package version 1.58.0. https://www.bioconductor.org/packages/release/bioc/html/impute.html. Accessed 13 Jan 2020

Hill DJ, Cameron DJ, Francis DE, Gonzalez-Andaya AM, Hosking CS (1995) Challenge confirmation of late-onset reactions to extensively hydrolyzed formulas in infants with multiple food protein intolerance. J Allergy Clin Immunol 96(3):386-394 [PubMed: 7560641]

Hyttinen JM, Niittykoski M, Salminen A, Kaarniranta K (2013) Maturation of autophagosomes and endosomes: a key role for Rab7. Biochim Biophys Acta 3:503-510

Jeffares DC, Penkett CJ, Bähler J (2008) Rapidly regulated genes are intron poor. Trends Genet 24:375-378 [PubMed: 18586348]

Kainulainen H, Hulmi JJ, Kujala UM (2013) Potential role of branched-chain amino acid catabolism in regulating fat oxidation. Exerc Sport Sci Rev 41(4):194-200 [PubMed: 23873132]

Kao M, Columbus DA, Suryawan A, Steinhoff-Wagner J, Hernandez-Garcia A, Nguyen HV, Fiorotto ML, Davis TA (2016) Enteral $\beta$-hydroxy- $\beta$-methylbutyrate supplementation increases protein synthesis in skeletal muscle of neonatal pigs. Am J Physiol Endocrinol Metab 310(11):E1072E1084 [PubMed: 27143558]

Kim SW, Wu G (2004) Dietary arginine supplementation enhances the growth of milk-fed young pigs. J Nutr 134:625-630 [PubMed: 14988458]

Kittler R, Putz G, Pelletier L, Poser I, Heninger AK, Drechsel D, Fischer S, Konstantinova I, Habermann B, Grabner H, Yaspo ML, Himmelbauer H, Korn B, Neugebauer K, Pisabarro MT, Buchholz F (2004) An endoribonuclease-prepared siRNA screen in human cells identifies genes essential for cell division. Nature 432(7020):1036-1040 [PubMed: 15616564] 
Kleinridders A, Pogoda HM, Irlenbusch S, Smyth N, Koncz C, Hammerschmidt M, Brüning JC (2009) PLRG1 is an essential regulator of cell proliferation and apoptosis during vertebrate development and tissue homeostasis. Mol Cell Biol 29(11):3173-3185 [PubMed: 19307306]

Kohlmeier M (2015) Nutrient metabolism: structures, functions, and genes. Academic Press, London

Krawiec BJ, Nystrom GJ, Frost RA, Jefferson LS, Lang CH (2007) AMP-activated protein kinase agonists increase mRNA content of the muscle specific ubiquitin ligases MAFbx and MuRF1 in C2C12 cells. Am J Physiol Endocrinol Metab 292:E1555-E1567 [PubMed: 17264220]

Lessard SJ, Rivas DA, So K, Koh HJ, Queiroz AL, Hirshman MF, Fielding RA, Goodyear LJ (2016) The AMPK-related kinase SNARK regulates muscle mass and myocyte survival. J Clin Investig 126(2):560-570 [PubMed: 26690705]

Manjarín R, Columbus DA, Suryawan A, Nguyen HV, Hernandez-García AD, Hoang NM, Fiorotto ML, Davis T (2016) Leucine supplementation of a chronically restricted protein and energy diet enhances mTOR pathway activation but not muscle protein synthesis in neonatal pigs. Amino Acids 48(1):257-267 [PubMed: 26334346]

Manjarín R, Columbus DA, Solis J, Hernandez-García AD, Suryawan A, Nguyen HV, McGuckin MM, Jimenez RT, Fiorotto ML, Davis TA (2018) Short- and long-term effects of leucine and branchedchain amino acid supplementation of a protein- and energy-reduced diet on muscle protein metabolism in neonatal pigs. Amino Acids 50(7):943-959 [PubMed: 29728917]

Mashako MN, Bernard C, Cezard JP, Chayvialle JA, Navarro J (1987) Effect of total parenteral nutrition, constant rate enteral nutrition, and discontinuous oral feeding on plasma cholecystokinin immunoreactivity in children. J Pediatr Gastroenterol Nutr 6(6):948-952 [PubMed: 3119814]

Mortazavi A, Williams BA, McCue K, Schaeffer L, Wold B (2008) Mapping and quantifying mammalian transcriptomes by RNA-Seq. Nat Methods 5:621-628 [PubMed: 18516045]

National Research Council (1998) Nutrient Requirements of Swine: 10th Revised Edition. The National Academies Press, Washington, DC

Pandya-Jones A (2011) Pre-mRNA splicing during transcription in the mammalian system. Wiley Interdiscip Rev RNA 2(5):700-717 [PubMed: 21823230]

Parker P, Stroop S, Greene H (1981) A controlled comparison of continuous versus intermittent feeding in the treatment of infants with intestinal disease. J Pediatr 99(3):360-364 [PubMed: 6790688]

Paulsen RD, Soni DV, Wollman R, Hahn AT, Yee MC, Guan A, Hesley JA, Miller SC, Cromwell EF, Solow-Cordero DE, Meyer T, Cimprich KA (2009) A genome-wide siRNA screen reveals diverse cellular processes and pathways that mediate genome stability. Mol Cell 35(2):228-239 [PubMed: 19647519]

Piepho HP (2009) Data transformation in statistical analysis of field trials with changing treatment variance. Agron J 101:865-869

Sandri M (2010) Autophagy in health and disease. 3. Involvement of autophagy in muscle atrophy. Am J Physiol Cell Physiol 298(6):C1291-C1297 [PubMed: 20089936]

Sandri M, Sandri C, Gilbert A, Skurk C, Calabria E, Picard A, Walsh K, Schiaffino S, Lecker SH, Goldberg AL (2004) Foxo transcription factors induce the atrophy-related ubiquitin ligase atrogin-1 and cause skeletal muscle atrophy. Cell 117:399-412 [PubMed: 15109499]

Shannon P, Markiel A, Ozier O, Baliga NS, Wang JT, Ramage D, Amin N, Schwikowski B, Ideker T (2003) Cytoscape: a software environment for integrated models of biomolecular interaction networks. Genome Res 13(11):2498-2504 [PubMed: 14597658]

Shulman RJ, Redel CA, Stathos TH (1994) Bolus versus continuous feedings stimulate small-intestinal growth and development in the newborn pig. J Pediatr Gastroenterol Nutr 18(3):350-354 [PubMed: 8057220]

Singh J, Padgett RA (2009) Rates of in situ transcription and splicing in large human genes. Nat Struct Mol Biol 16:1128-1133 [PubMed: 19820712]

Stitt TN, Drujan D, Clarke BA, Panaro F, Timofeyva Y, Kline WO, Gonzalez M, Yancopoulos GD, Glass DJ (2004) The IGF-1/PI3K/Akt pathway prevents expression of muscle atrophy-induced ubiquitin ligases by inhibiting FOXO transcription factors. Mol Cell 14:395-403 [PubMed: 15125842] 
Suryawan A, Rudar M, Fiorotto ML, Davis TA (2020) Differential regulation of mTORC1 activation by leucine and $\beta$-hydroxy- $\beta$-methylbutyrate in skeletal muscle of neonatal pigs. J Appl Physiol 128(2):286-295 [PubMed: 31944890]

Tannous RE, Rogers QR, Harper AE (1963) Effect of leucine-isoleucine and valine antagonism on the pattern of free amino acids in blood plasma and several tissues of the rat. Fed Proc 22:202-210

The UniProt Consortium (2019) UniProt: a worldwide hub of protein knowledge. Nucleic Acids Res 47:D506-D515 [PubMed: 30395287]

Tooze SA, Abada A, Elazar Z (2014) Endocytosis and autophagy: exploitation or cooperation? Cold Spring Harb Perspect Biol 6(5):a018358 [PubMed: 24789822]

Torrazza RM, Suryawan A, Gazzaneo MC, Orellana RA, Frank JW, Nguyen HV, Fiorotto ML, El-Kadi S, Davis TA (2010) Leucine supplementation of a low-protein meal increases skeletal muscle and visceral tissue protein synthesis in neonatal pigs by stimulating mTOR-dependent translation initiation. J Nutr 140(12):2145-2152 [PubMed: 20962152]

Troyanskaya O, Cantor M, Sherlock G, Brown P, Hastie T, Tibshirani R, Botstein D, Altman RB (2001) Missing value estimation methods for DNA microarrays. Bioinformatics 17(6):520-525 [PubMed: 11395428]

Wei B, Dui W, Liu D, Xing Y, Yuan Z, Ji G (2013) MST1, a key player, in enhancing fast skeletal muscle atrophy. BMC Biol 11:12 [PubMed: 23374633]

Wheatley SM, El-Kadi SW, Suryawan A, Boutry C, Orellana RA, Nguyen HV, Davis SR, Davis TA (2014) Protein synthesis in skeletal muscle of neonatal pigs is enhanced by administration of $\beta$ hydroxy- $\beta$-methylbutyrate. Am J Physiol Endocrinol Metab 306:E91-E99 [PubMed: 24192287]

Wickramasinghe S, Rincon G, Islas-Trejo A, Medrano JF (2012) Transcriptional profiling of bovine milk using RNA sequencing. BMC Genomics 13:45 [PubMed: 22276848]

Wilson FA, Suryawan A, Gazzaneo MC, Orellana RA, Nguyen HV, Davis TA (2010) Stimulation of muscle protein synthesis by prolonged parenteral infusion of leucine is dependent on amino acid availability in neonatal pigs. J Nutr 140:264-270 [PubMed: 20032489]

Yoshii SR, Mizushima N (2017) Monitoring and measuring autophagy. Int J Mol Sci 18(9):E1865 [PubMed: 28846632] 
(a)

$$
\begin{array}{r}
\text { Age (d) } \\
\text { Study (d) } \\
\text { Weaning } \\
\text { Bowl feeding } \\
\text { Catheter placement } \\
\text { Orogastric Feeding } \\
\text { Leu/Ala infusion } \\
\text { Blood metabolomics } \\
\text { Muscle Western Blot } \\
\text { Muscle RNA-seq }
\end{array}
$$

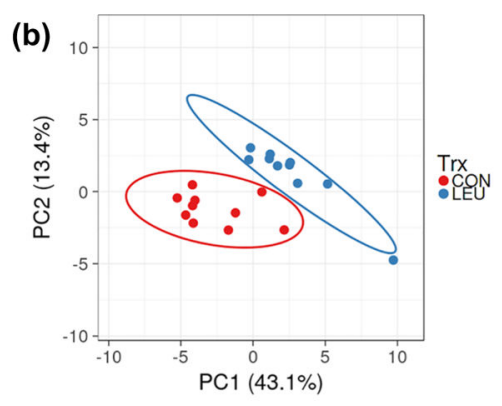

(c)
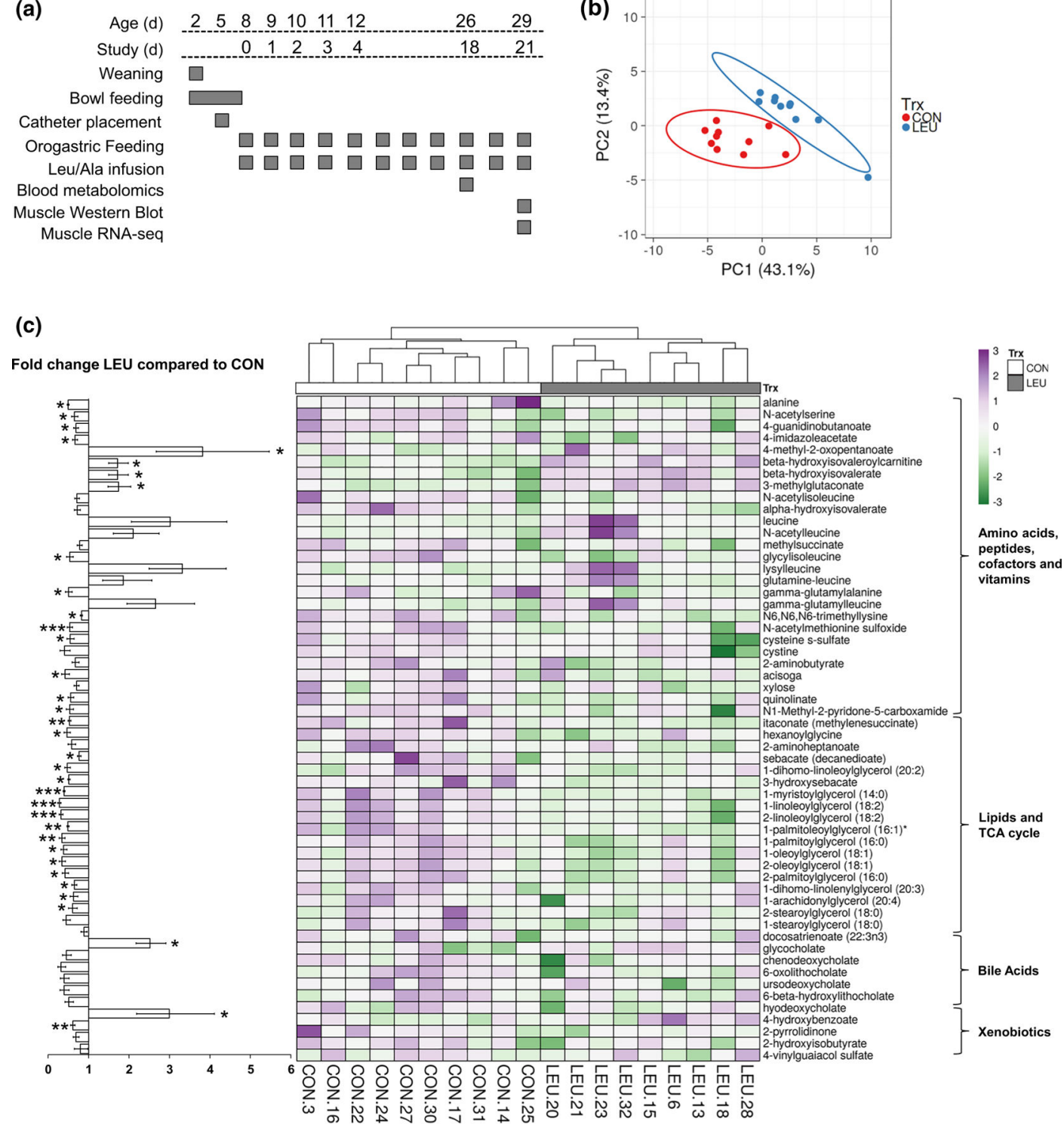

Fig. 1.

a Administration of intermittent leucine pulses in continuously orogastric-fed pigs altered the metabolomic profile in plasma. Outline of the experimental design of the animal study. Twenty-three 8-day-old piglets were assigned to receive either leucine (LEU, $n=11$ ) or alanine (CON, $n=12$ ) pulses for $1 \mathrm{~h}$ every $4 \mathrm{~h}$ for 21 days. At the same time, all pigs were fed continuously a liquid diet. b Principal component analyses of metabolites discriminated LEU- and CON pigs along with the first component. Data were scaled to unit variance prior to PC assessment. Each point represents an individual pig and color of point denotes diet. c Heat map of relative abundance of metabolites significantly changed in neonatal pigs, with fold change and significance levels by LEU compared to CON. Columns are individual pigs and rows are log-transformed metabolite data. Violet and green represent the row minimum and maximum values. $P$ values for each metabolite were calculated by a one-way ANOVA 
with a mixed model. Significant $P$ values are expressed as ${ }^{\dagger} P \leq 0.1, * P \leq 0.05$, ** $P \leq 0.01$, $* * * P \leq 0.001$ 


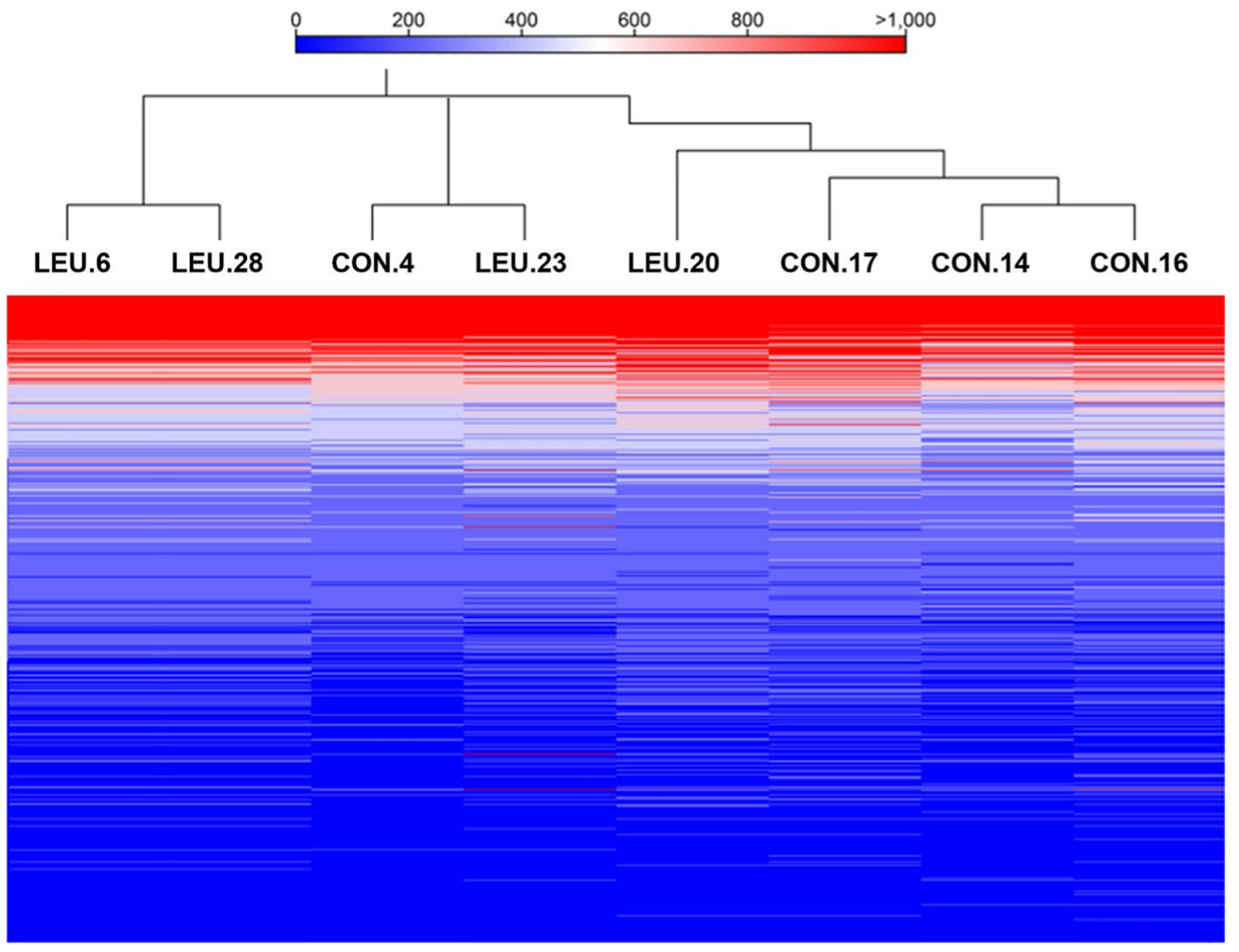

Fig. 2.

Administration of intermittent leucine pulses in continuously orogastric-fed pigs altered the transcriptome profile in skeletal muscle. LD samples from four CON and four LEU pigs representative of the average body weight gain for each group were used for RNA isolation and sequencing. Hierarchical cluster analysis was performed to validate the treatment distribution using the normalized mean expression of the whole transcriptome across the eight libraries. Red: highly expressed genes; blue: lower expressed genes; white: medium expressed genes 

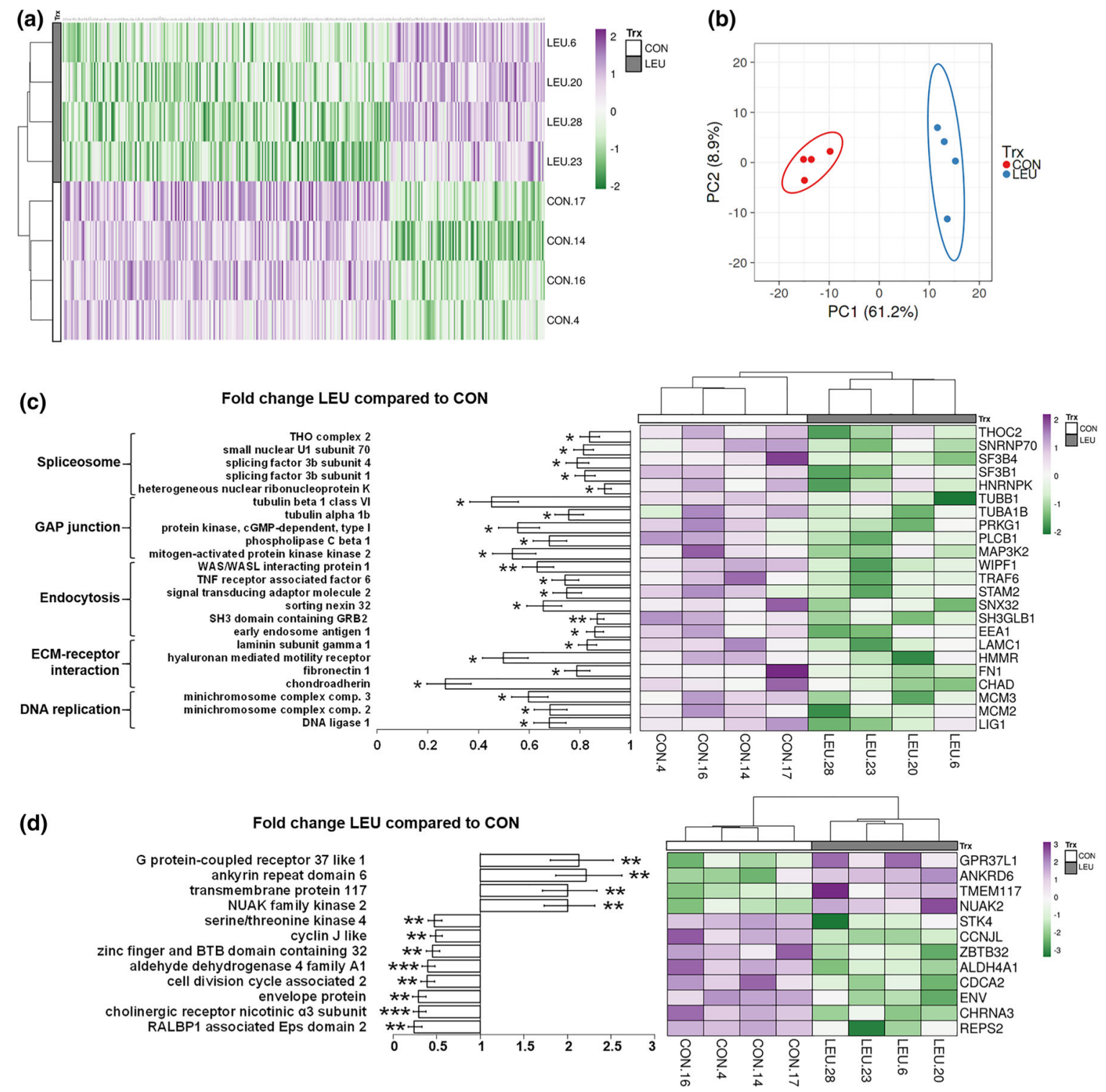

Fig. 3.

a Heat map and principal component analysis of differentially expressed genes (DEGs) of skeletal muscle samples from neonatal pigs fed a liquid diet continuously via orogastric tube and supplemented with 1-h parenteral pulses of leucine (LEU; $n=4)$ or alanine (CON, $n=$ 4) every $4 \mathrm{~h}$ for 21 days. Columns are $\log _{2}$-transformed reads per kilobase per million mapped reads (RPKMs) and rows are individual pigs. Violet and green represent the column minimum and maximum values, respectively. b Principal component analyses of skeletal muscle samples using the top 465 genes separated LEU and CON pigs into two different clusters along with the first component. Each point represents an individual pig and color of point denotes diet. $\mathbf{c}$ Heat map of groups of genes selected among the top 465 DEGs sharing common Kyoto Encyclopedia of Genes and Genomes (KEGG) terms, with fold change and significance levels by LEU compared to CON. $\mathbf{d}$ Heat map of DEGs selected using the most stringent parameters (RPKM $P \leq 0.01,+$ fold change $>2$ ), with fold change and significance 
levels for LEU compared to CON. In all heat maps violet and green represent the row minimum and maximum values, respectively. Columns are individual pigs and rows are $\log _{2}$-transformed RPKMs. $P$ values for each gene were calculated by a one-way ANOVA with a mixed model. Significant $P$ values are expressed as $* P \leq 0.05$, $* * P \leq 0.01, * * * P \leq$ 0.001 


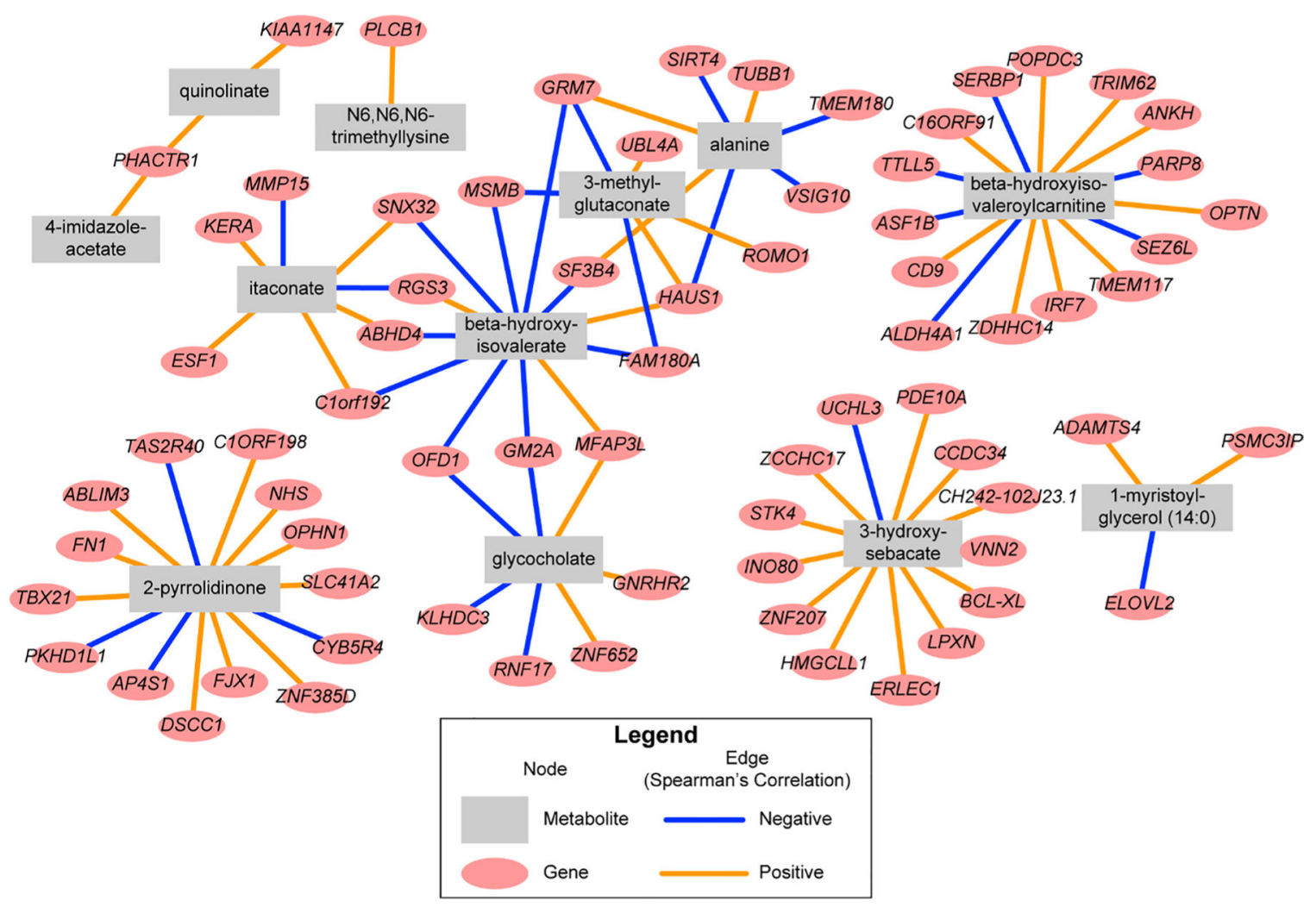

Fig. 4.

Plasma metabolites were correlated with transcriptome changes in skeletal muscle.

Associations between muscle mRNA sequencing (RNA-seq) and plasma untargeted metabolomics in neonatal pigs fed continuously with an orogastric tube for 21 days and supplemented with either leucine or alanine pulses parenterally at 4-h intervals. RNA-seq data are reads per kilobase per million mapped reads. Metabolomics data are logtransformed peak values. Spearman's correlations were assessed on genes and metabolites previously shown to be altered by leucine. Results were adjusted for multiple comparisons using Benjamin and Hochberg's false discovery rate correction (FDR). Only correlations at FDR $<0.1$ are described in network. Genes are red ovals; metabolites are rectangles. Positive correlations are shown as orange edges; negative correlations are blue edges 


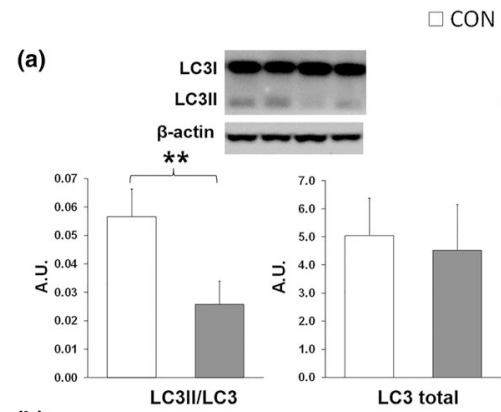

(b)
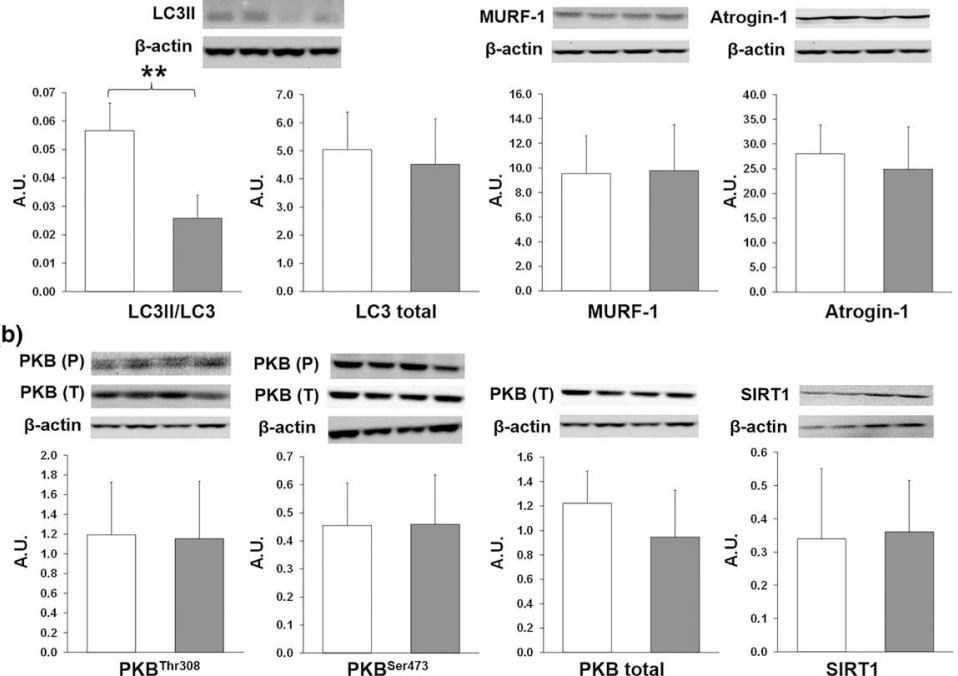

(c)
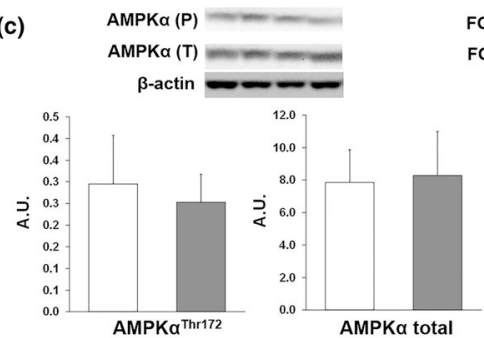

FOXO1 (P) $--\ldots-$ FOXO3 (P) - FOX01 (T) $=-\ldots$ FOXO3 (T) $\ldots=-$

(d) STK4 (P) STK4 (T) - -

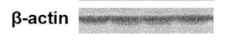
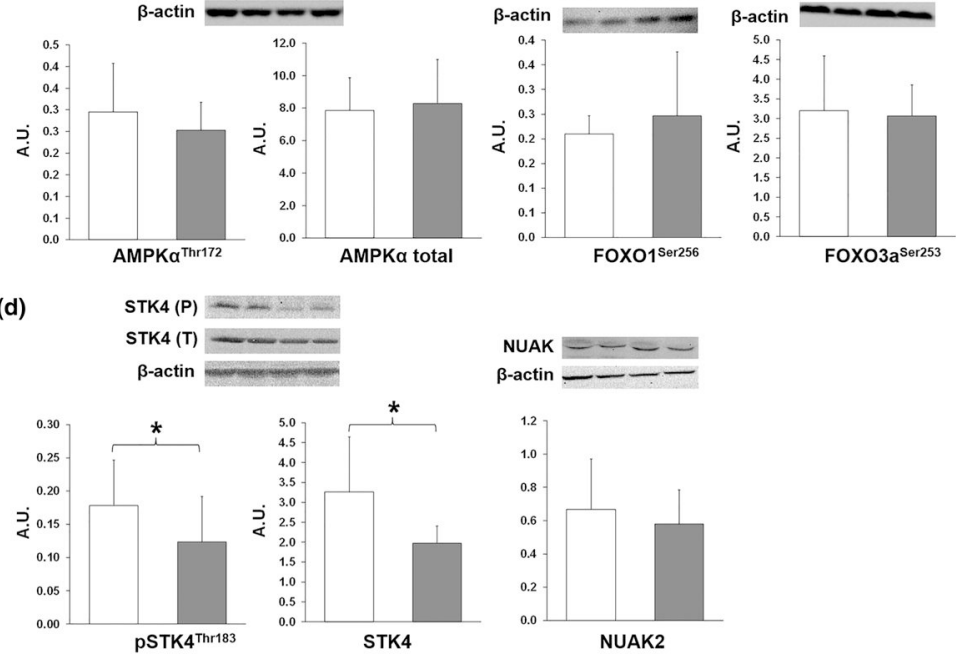

Fig. 5.

Markers of protein degradation decreased in response to leucine (LEU, $n=11$ ) or alanine (CON, $n=12$ ) pulses in neonatal pigs continuously fed formula via orogastric tube for 21 days. Representative Western blots (top) and histograms (bottom) with the quantification of bands expressed as arbitrary units (A.U.), measuring the abundance and phosphorylation of microtubule-associated protein 1A/1B light chain 3A (LC3I/II), Atrophy F-Box/Atrogin-1 (MAFbx/atrogin-1) and muscle RING-finger protein-1 (MuRF1) (a), protein kinase B (PKB) and sirtuin 1 (SIRT-1) (b), AMP-activated protein kinase a (AMPKa), forkhead box O1 (FOXO1) and FOXO3a (c), and serine/threonine kinase 4 (STK4) and carbon catabolitederepressing protein kinase-AMPK-related kinase (NUAK2) (d). Values are means $\pm \mathrm{SD} . * P$ $\leq 0.05 * * P \leq 0.01$ 


\section{Table 1}

Ingredient composition $(\%)$ of diets fed to CON (control + alanine pulses; $n=12)$ and LEU (control diet + leucine pulses; $n=11$ ) pigs between day 0 and 21 of the study

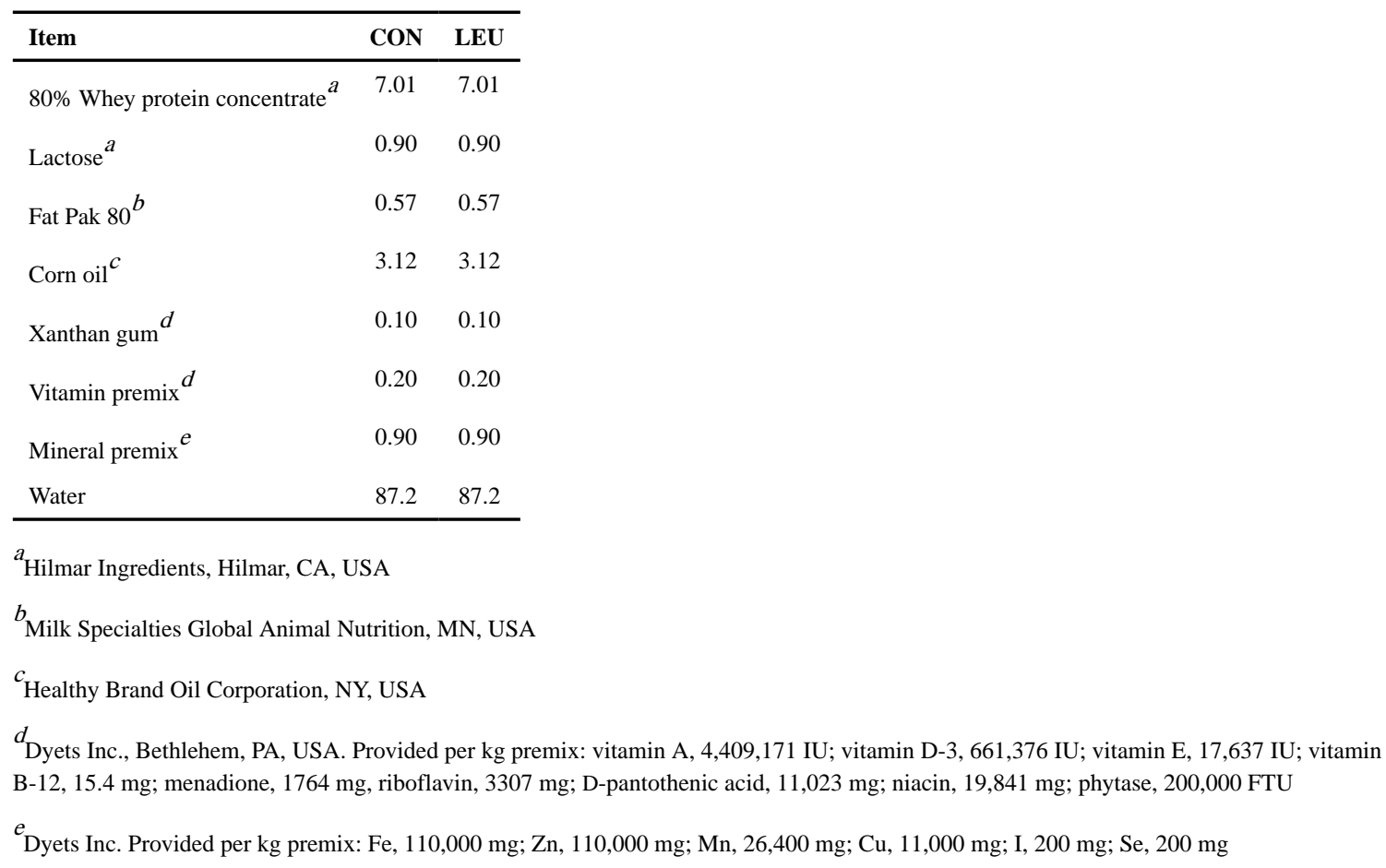




\section{Table 2}

Daily nutrient ( $\mathrm{g} \mathrm{kg}$ body weight $(\mathrm{BW})^{-1}$ day $^{-1}$ ) and metabolizable energy (ME; $\mathrm{kcal} \mathrm{kg} \mathrm{BW} \mathrm{BW}^{-1} \mathrm{day}^{-1}$ ) fed to CON (control + alanine pulses; $n=12$ ) and LEU (control + leucine pulses; $n=11$ ) pigs between day 0 and 21 of the study. Values are calculated, and expressed as fed

\begin{tabular}{lcc}
\hline Item $\left(\mathbf{k g ~ B W} \mathbf{~}^{\mathbf{1}} \mathbf{d a y}^{\mathbf{- 1}}\right.$ ) & CON & LEU \\
\hline Dry matter $(\mathrm{g})$ & 22.5 & 22.5 \\
Crude protein (g) & 10.1 & 10.1 \\
Metabolizable energy (kcal) & 116.6 & 116.6 \\
Carbohydrates (g) & 3.32 & 3.32 \\
Ether extract (g) & 7.03 & 7.03 \\
Amino acids (g) & & \\
Arginine & 0.23 & 0.23 \\
Histidine & 0.20 & 0.20 \\
Isoleucine & 0.60 & 0.60 \\
Leucine & 1.11 & 1.11 \\
Lysine & 0.85 & 0.85 \\
Methionine & 0.20 & 0.20 \\
Cysteine & 0.25 & 0.25 \\
Phenylalanine & 0.31 & 0.31 \\
Tyrosine & 0.24 & 0.24 \\
Threonine & 0.58 & 0.58 \\
Tryptophan & 0.15 & 0.15 \\
Valine & 0.53 & 0.53 \\
Calcium (g) & 0.62 & 0.62 \\
Phosphorus (g) & 0.43 & 0.43 \\
\hline
\end{tabular}




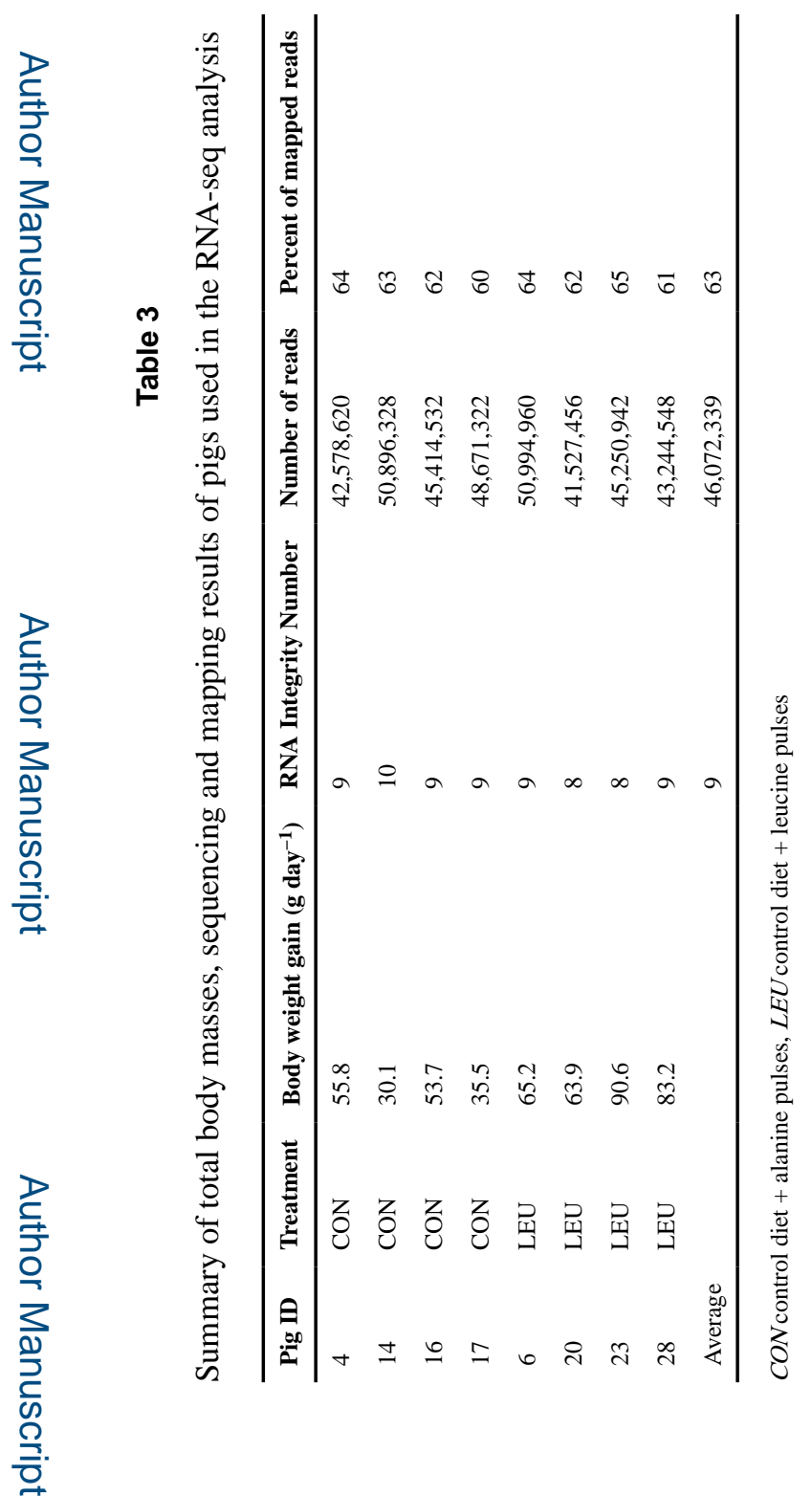

Amino Acids. Author manuscript; available in PMC 2021 September 24. 


\section{옴 \\ \)

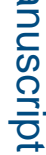

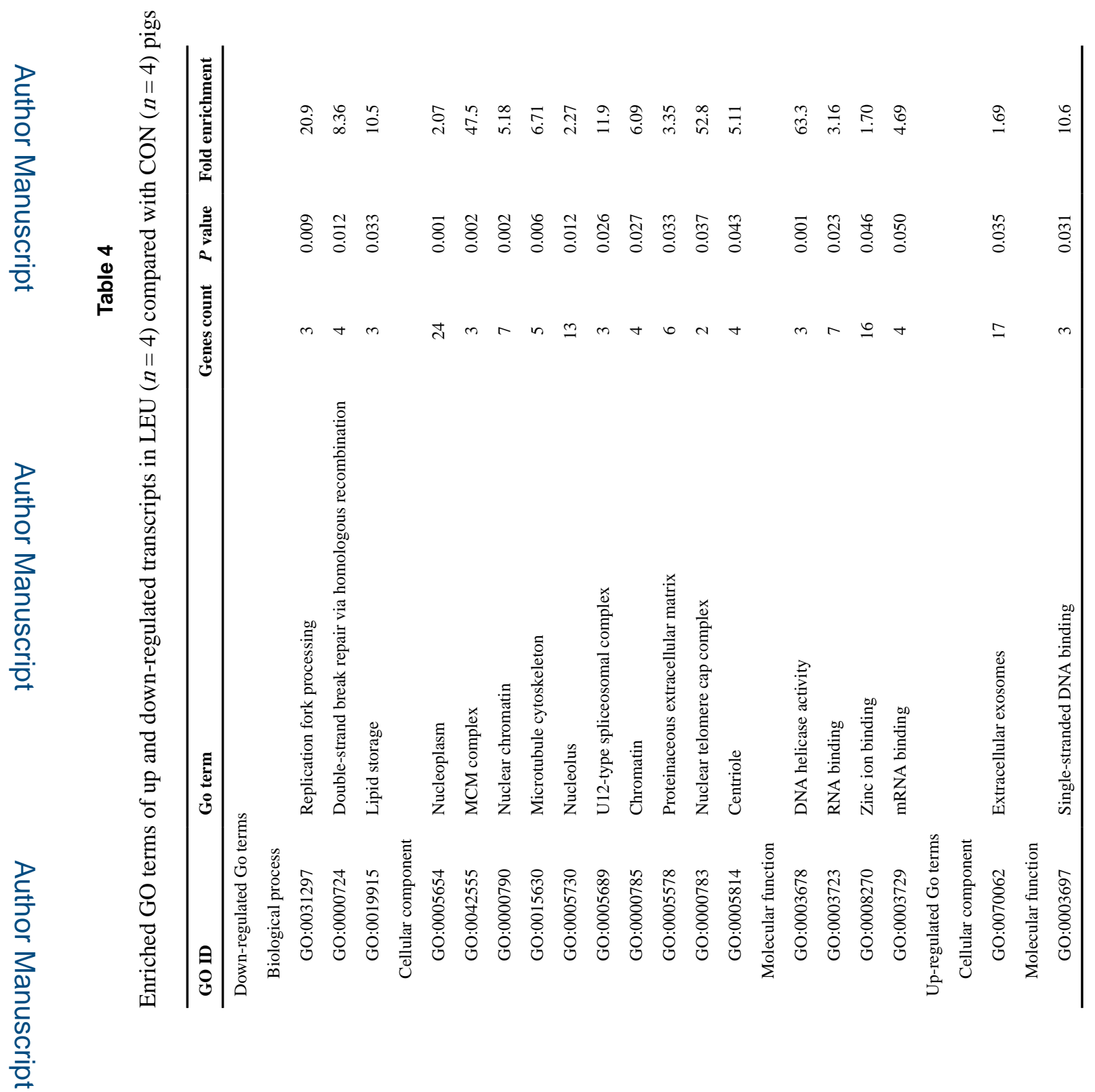

Amino Acids. Author manuscript; available in PMC 2021 September 24. 